\title{
Lo que vibra es el yunque: Análisis de Nicom. Harm. VI, pp. 245.18-248.26
}

\author{
Fuensanta GARRIDO DOMENÉ \\ Universidad de Huelva \\ fuengarrido@gmail.com
}

Recibido: $13-9-2011$

Aceptado: 14-11-2011

\section{RESUMEN}

La primera y más antigua versión de la leyenda de los martillos pitagóricos en su forma completa fue redactada por Nicómaco de Gerasa, un músico y matemático neopitagórico. En ella, se describen las distintas relaciones numéricas que definen los intervalos musicales, lo que permite un análisis de estilo y de contenido del todo original: de estilo, porque éste cambia respecto a los demás capítulos del tratado musical nicomaqueo en virtud de un fin claramente didáctico, y de contenido porque, en la minuciosa descripción de los distintos pasos del experimento pitagórico llevado a cabo con los martillos y los pesos en los extremos de las cuerdas, se entreven unas conclusiones que no son del todo exactas ni para la tensión ni para la percusión.

Palabras clave: Nicómaco de Gerasa, Pitágoras, música griega antigua, pitagorismo y neopitagorismo, leyenda pitagórica.

\begin{abstract}
The first and the most ancient complete version of the legend of pythagorean hammers was written by Nicomachus of Gerasa, a neopythagorean musician and mathematician. The different numerical proportions which delimit the musical intervals are described in this passage. It allows to analize the style and the content of this chapter: the style, because it is different with regard to the other chapters of the manual on account of its didactic purpose; and the content because, in the description of the pythagorean experiment with hammers and the weights hanging in the extremes of the strings, conclusions not precise for tension or for percussion are sensed.
\end{abstract}

Key words: Nicomachus of Gerasa, Pythagoras, ancient greek music, pythagoreanism and neopythagoreanism, pythagorean legend.

1. Lo que vibra es el yunque. Pero, ¿qué yunque y en qué contexto? El yunque es el del herrero y el contexto es, evidentemente, el de la herrería. Eminentes cálamos y plumas de la época antigua y tardoantigua han legado al mundo de la filología y de la musicología la célebre historia del descubrimiento, por parte de Pitágoras, de las relaciones numéricas que definen los intervalos musicales. Adrasto, Gaudencio el Filósofo, Arístides Quintiliano, Claudio Tolomeo, Porfirio, Diógenes Laercio, Jámblico de 
Calcis, Censorino, Calcidio, Macrobio, Fulgencio, Boecio, Isidoro de Sevilla e incluso Guido d'Arezzo se cuentan entre ellos ${ }^{1}$.

Ante tal abundancia de escritos de esta índole, cabe preguntarse cuál es su fuente originaria $\mathrm{o}$, al menos, de quién la oyeron o la leyeron por vez primera.

2. La crítica se aúna para reconocer como la primera y más antigua versión de la leyenda de los martillos pitagóricos en su forma completa la que ofrece el neopitagórico Nicómaco de Gerasa (ca. 80 a. C. - ca. 140 ó 150 d. C.) en el capítulo VI de su

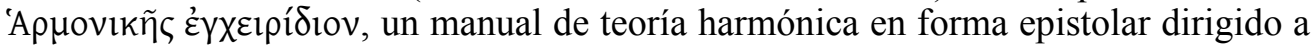
una dama anónima ${ }^{2}$. Este tratado es, junto con el pseudo-aristotélico Problemata, la Sectio canonis y el pseudo-plutarqueo De musica, el único escrito completo que existe entre el periodo que va desde las obras harmónicas de Aristóxeno de Tarento y de Claudio Tolomeo. Algunos estudiosos ${ }^{3}$ se aventuran, incluso, a calificar a la destinataria del tratado como la primera persona en la historia documentada en leer este relato. Si ello fuera así, habría que aceptar que el de Gerasa contó a su discípula la anécdota de Pitágoras y el herrero «armonioso» ${ }^{4}$ precisamente en la misma forma en la que él la escuchó, sin ninguna modificación y, por supuesto, sin intención alguna de transmitir sus improbabilidades y/o imprecisiones. Este mismo propósito se manifiesta en la labor de difusión de los eruditos antiguos, quienes, como él, se afanaron en conservar y propagar, con sorprendentes correspondencias ad verbum, la mismísima versión nicomaquea, aun siendo conscientes de sus incongruencias.

3. Hay dos interrogantes que se ciernen sobre el propio relato: ¿es real la atribución a Pitágoras de estos descubrimientos acústicos o se trata de una adaptación pitagórica de otro relato?; si es así, ¿cuál fue la fuente de Nicómaco?

Frente a aquéllos que sostienen el genio de Pitágoras en este descubrimiento o, al menos, admiten una muy posible suposición, se alza la voz de los que, por el contrario, dudan al respecto, o incluso lo niegan ${ }^{5}$. De los defensores antiguos de la autoría del de Samos es menester considerar también la postura que hace suponer a Pitágoras como el primer hombre que descubrió ( $\pi \rho \tilde{\omega} \tau o \zeta ~ \alpha ̉ v \varepsilon v \rho \eta \kappa \varepsilon ́ v \alpha ı ~ \delta o к \varepsilon \tilde{)})$ tales proporcio-

${ }^{1} A p$. Theo Sm. LVI 9-12; Gaud. Harm. XI, Jan:340.4-341.11; Aristid. Quint. III 1, W-I:94.11-95.7; Ptol. Harm. I 3, Düring:6.14-9.15; Porph. In Harm. 119.29-120.7 Düring; D.L. VIII 12 Long; Iamb. VP. 115-120; Cens. X; Chalc. Tim. XLV, Waszink:93-94; Macr. In Somn. II 1.8-14; Fulg. Mythologiae, III 9, Helm:75; Boet. Mus. I 10-11; Guido Aretinus, Micrologus, XX, respectivamente.

${ }^{2}$ Harm. VI, Jan:245.18-248.26. En cuanto a la identificación de la destinataria del escrito, se han postulado tres posibles respuestas: una pitagórica, teoría que se fundamenta en la lista de miembros femeninos aceptada por los componentes de la secta (Iamb. VP. 267.66-77 Deubner); una dama innominada; o la emperatriz Plotina Augusta, esposa del emperador Trajano. Vid. Ruelle (1880:10, n. 2), D’Ooge et alii (1926:76-77), McDermott (1977) y LeVIN (1975:17-18).

${ }^{3}$ LeVIN (1994:86).

${ }^{4}$ Georg Friederich HäNDEL (1685-1759) recogió la fábula de Pitágoras y los martillos de una herrería a la manera de motivo musical en el Air con Variazioni de Harpsichord Suite No. V, un movimiento conocido comúnmente como «El herrero armónico».

${ }^{5} C f$. Levin (1975:67-74) para un detallado repaso de sendas posturas, tanto en la crítica moderna como en la antigua. 
nes harmónicas ${ }^{6}$. Del mismo modo, no ha de pasarse por alto la opinión de ciertas autoridades de la Antigüedad, como Aristóteles o Teón de Esmirna, en cuyos escritos acerca de este episodio los protagonistas son, si acaso, los pitagóricos ${ }^{7}$. Noticias como éstas desembocan en una incapacidad para asegurar si el descubrimiento fue hecho por Pitágoras, por los pitagóricos o, como dice Arístides Quintiliano, por «los primeros sabios $»^{8}$. Andrew Barker 9 , por su parte, señala a los propios artistas musicales o a los fabricantes de instrumentos como los descubridores de estas proporciones harmónicas, alegando que, con toda probabilidad, habrían hallado este suceso como algo útil en la construcción de liras y otros cordófonos, instrumentos populares en Grecia durante el siglo vi a. C. y cuya generalización en las culturas orientales mediterráneas remonta mucho antes.

A pesar del interés de Pitágoras por la aritmética ${ }^{10} \mathrm{y}$ a pesar del peso de la tradición, que le asigna la determinación matemática de los intervalos de cuarta, quinta y octava, lo cierto es que no hay ningún testimonio definitivo anterior al de Nicómaco que confirme esta afirmación ${ }^{11}$, lo que hace de nuestro autor la fuente para la «historia», digamos, «apócrifa». Sin embargo, aunque él no fue su inventor, contamos con una noticia dada a conocer por Porfirio sobre la actividad musical del maestro de Samos. En un

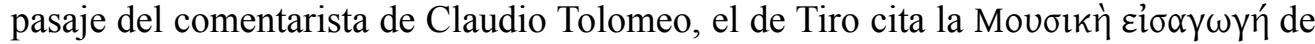
un tal Heraclides, que a su vez cita a Jenócrates, transmisor del testimonio más antiguo que atribuye el descubrimiento de las proporciones harmónicas de los intervalos musicales al propio Pitágoras ${ }^{12}$.

4. La fábula de los martillos y los pesos otorga al tratado nicomaqueo un carácter arcaico y una reflexión sobre la música del pasado, además de invitar al estudioso a analizar su peculiar estilo en tanto que éste es distinto respecto al de los restantes capítulos del manual. Frente al tecnicismo musical, matemático y astronómico mezclado, incluso, con la inclusión del dialecto dorio en el capítulo IX, el completo cambio en la narración de este epígrafe parece dar fe de una intención aclaratoria y facilitadora de lo que sigue. Si se presta atención al texto griego, se verán, además, los fundamentos en que se apoya la crítica para ver un «cuento» en el relato del experimento con los martillos. Así, se reconocen ${ }^{13}$ en él elementos propios del mito y/o de la leyenda

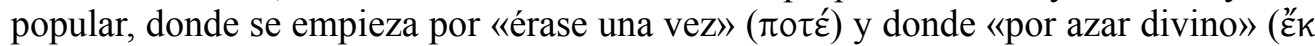

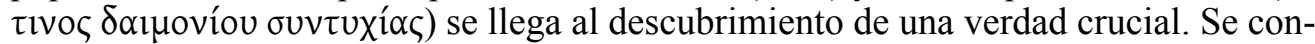
sideran, por tanto, unos antecedentes muy antiguos y de tono oriental relacionados, por supuesto, con los orígenes de la música. Ello reafirma la idea, convertida ya en

${ }^{6} \mathrm{Cf}$. Adrasto ap.Theo Sm. LVI 9-12.

${ }^{7}$ Cf. Arist. Metaph. 985b23-986a13, en cuyas líneas dedicadas al pensamiento de los pitagóricos y de los eléatas, el filósofo de Estagira dice que aquéllos veían en los números las proporciones y propiedades de las harmonías musicales; y Theo Sm. LIX 4-21.

${ }^{8}$ III 1, W-I:94.11-95.7.

${ }^{9}$ (1989:256, n. 43).

${ }^{10}$ Cf. Aristox. Fr. 23 Wehrli.

${ }^{11}$ Philip (1966:125).

${ }^{12}$ In Harm. 30.1-9. Vid. Meriani (1995) y Redondo Reyes (2005).

${ }^{13}$ LeVin (1975:69-74). De la misma opinión son Burnet (1957:107) y ZelLer (19196:370). 
afirmación, de que una persona de habla no griega registró la historia por escrito antes que Nicómaco. Estaríamos, por tanto, ante la conexión de la versión pitagórica con una versión existente completamente aparte de la tradición seguidora del maestro de Samos. Pese a que se ha estimado ${ }^{14}$ que el nombre de Pitágoras, en relación con las teorías elementales del número, es más que legendario y, por supuesto, está carente de valor histórico, sin embargo las semejanzas fehacientes de ambas «leyendas» evidencian que la concepción pitagórica de la relación entre música y número es asiática ${ }^{15}$.

Los propios autores antiguos ya aceptaron este hecho, pues Jámblico afirma que el descubrimiento de las proporciones harmónicas es propio de los babilónicos, de quien Pitágoras las aprendió y las llevó a Grecia ${ }^{16}$. Este mérito babilónico, de hecho, se ve reforzado por el empeño de sus matemáticos en determinar la misma irracionalidad de $\sqrt{2}$, implícita en la proporción epogdoica o de tono $(9: 8)$. Con todo, es difícil entender la conexión que hay entre $\sqrt{2}$ y $9: 8$, ya que no existe ninguna relación que se base en cálculos matemáticos. Se ha conjeturado que, con toda probabilidad, Pitágoras se basó en los conocimientos babilónicos ${ }^{17}$, primera civilización que utilizó un algoritmo para calcular el valor de los números radicales, si bien es cierto que el término «raíz» y su símbolo no se usaron hasta 1525, introducidos por el matemático alemán Christoff Rudolff (1499-1545).

Esta afirmación no puede ser ratificada en cuanto a la relación epogdoica, puesto que las tablas babilónicas de YBC 7289 (ca. 2000-1650 a.C.) proporcionan una aproximación de $\sqrt{2}$ en lo que es similar a seis cifras decimales (imagen 1).

Si se toman los dos primeros sumandos de esta operación (imagen 2) se obtendrá la proporción que frecuentemente utilizaba dicha civilización para aproximar el valor de $\sqrt{2}$, a saber, la relación epítrita $3: 4$ y no la epogdoica 9:8.

No obstante, podemos pensar en la irracionalidad de $\sqrt{2}$ como «implícita» en la proporción de tono en cuanto a su cálculo como diferencia entre las relaciones hemiólica y epítrita (imagen 3).

Al margen de la tradición babilónica, Porfirio enlaza a Pitágoras con los «dáctilos ideos» de $\mathrm{Creta}^{18}$, unos herreros diminutos que, según las crónicas de la antigua Frigia, eran maestros de las artes y de la artesanía, sirvientes de la diosa asiática Rea Cíbele, conocida por los romanos como Magna Mater, y responsables del descubrimiento de las bases matemáticas de la música en las diferentes notas de sus yunques. Isidoro de Sevilla ${ }^{19}$, a su vez, contempla una tradición perteneciente al Antiguo Testamento según la cual Jubal, un descendiente de Caín, fuit pater canentium cithara et organo ${ }^{20}$.

${ }^{14} C f$. Neugebauer (1957:35-36).

${ }^{15} C f$. Werner (1959:376-377).

${ }^{16}$ In Nic. 118.19-24.

${ }^{17}$ Aunque se denomine «método babilónico» de manera general, no existe evidencia que muestre un uso de esta aproximación por los babilónicos en el cálculo de $\sqrt{2}$, tal y como se puede ver en la tablilla YBC 7289. $C f$. FowLER-ROBSON (1998:376) y FLANNERY (2005:32 y 158).

${ }^{18}$ VP. XVII.

${ }^{19}$ Etym. III 16.1.

${ }^{20} \mathrm{Ge} 4.21$. 
Así las cosas, Nicómaco pudo conocer este vínculo entre un material de origen asiático y el nombre de Pitágoras y pudo, con toda probabilidad, manejarlo en fuentes ahora perdidas para plasmarlo en su tratado.

5. Del mismo modo, sea real o no la descripción que Nicómaco ofrece de los martillos, lo que sí que es cierto — y esto lo sabemos por Jámblico — es que Pitágoras fue un músico soberbio, dado que fue el primero en reconocer el valor terapéutico de la música y los efectos éticos que produce sobre el $\mathrm{alma}^{21}$. Su preferencia por la lira frente al auló, por ser aquélla un instrumento especialmente apropiado para acompañar los himnos a los dioses, nos es igualmente conocida gracias a Diógenes Laercio ${ }^{22}$. De ahí que se haya pensado en su atención fijada en la lira y en las diversas posibilidades de afinación hasta llegar a lo que Nicómaco describió en el capítulo V del Manual de harmónica, id est, el octacordio dorio ${ }^{23}$. Así, para demostrar que en su lograda escala sus dos nuevas quintas eran idénticas y que, a su vez, sus dos nuevas cuartas, también iguales, estaban separadas por un tono, se valió únicamente de dos «instrumentos»:

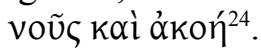

Con ellos, según el texto, Pitágoras estaba buscando un método objetivo para definir, de la manera más exacta posible, las distintas notas. Logró su empeño de manera

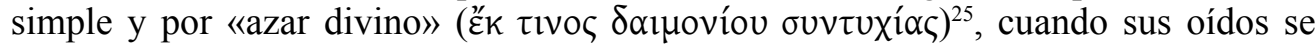
llenaron con el efecto que producía el sonido procedente de diferentes martillos de una herrería al chocar con el yunque. Como cuenta el geraseno, Pitágoras reconoció entre estos sonidos un intervalo de cuarta, otro de quinta y un último de octava en proporciones idénticas a los que producía en las cuerdas de su lira (imagen 4). A partir de ahí, se dio cuenta de que estos intervalos eran consonantes y agradables - esto

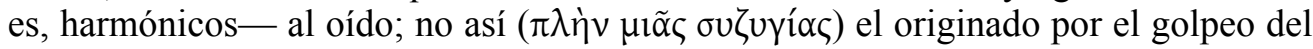
martillo que generaba la cuarta junto con el que generaba la quinta, a saber, la combinación ( $\left.\sigma \cup \zeta \cup \gamma^{\prime} \alpha\right)$ marcada por el tono introducido por él entre los dos tetracordios de su original lira heptacorde ${ }^{26}$.

6. Para Pitágoras, el conocimiento de la verdad absoluta se limita al saber de las leyes universales que gobiernan toda realidad. En el caso de la harmonía, la verdad era el principio según el cual se construía la $\alpha \rho \mu o v i ́ \alpha$ doria $^{27}$. El principal medio para

${ }^{21}$ Cf. Iamb. VP. 35.110.

${ }^{22}$ VIII 24.

${ }^{23} C f$. Levin (1994:86-97). Aunque esta escala es la misma que aparece en la División del canon de Euclides, ha sido considerada como mera construcción metafísica por parte de Frank (1923:166ss.) y MACDONALD CORNFord $(1937: 67,68,72)$, entre otros.

${ }^{24} C f$. Iamb. VP. 32.228, según el cual Pitágoras enseñó a sus discípulos a hacer lo mismo.

${ }^{25}$ Esta expresión la volvemos a encontrar en los diversos relatos de lo que hemos llamado «los

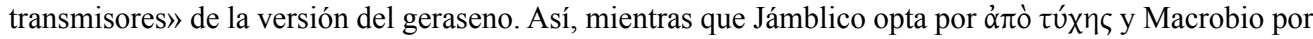

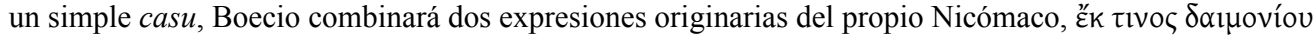

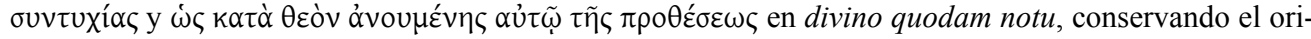
gen científico y divino de la música para los autores cristianos venideros.

${ }^{26}$ En otras palabras, el intervalo que hace la diferencia entre la cuarta y la quinta.

${ }^{27}$ La harmonía doria fue aceptada, de manera unánime, como el octacordio ( $c f$. Pl. La. 188d, donde

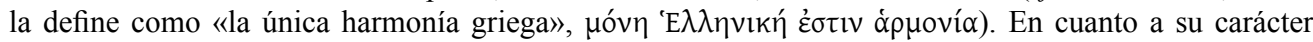


llegar a él y proporcionar, así, una prueba científicamente fiable es la mente, pues ella es «la que ve y escucha todo $\rangle^{28}$ pudiendo, de este modo, ser provista de todo tipo de información exacta. Sin embargo, y pese a que los sentidos no son aptos para hallar tales descubrimientos, éstos favorecerán y ampararán la «instrumentalidad» de aquélla. Recurriendo al procedimiento de la comparación, Nicómaco recoge en estas líneas cuáles son las herramientas con las que cuentan los sentidos de la vista y del tacto para alcanzar con éxito una investigación precisa y verdadera. Así, entre los instrumentos «de

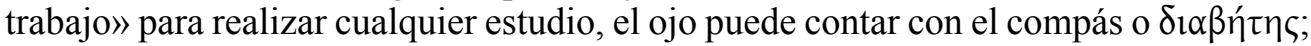

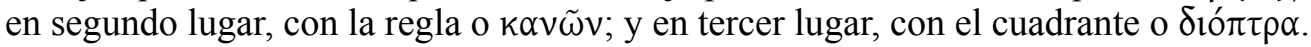
El tacto tampoco está falto de ayuda en su labor de calcular el peso justo de las cosas, ya que cuenta con la balanza, peso simple o לuyóc. Algunos de estos instrumentos ya fueron mencionados por Platón en un pasaje en el que declara la prácticamente nula exactitud y sistematización del arte de la música frente a todas las demás ${ }^{29}$.

Con la mención de estos útiles Nicómaco muestra y demuestra la incapacidad del oído para realizar estimaciones del tamaño de un intervalo a otro sin una «ayuda instrumental $»^{30}$. La inexistencia de una herramienta auditiva para lograr determinar la diferencia entre tonos y conseguir, así, una afinación medianamente «decente» fue motivo de burla por parte de Glaucón en el diálogo platónico en el que éste participa ${ }^{31}$.

7. Sea como fuere, descubriera Pitágoras por sí mismo la verdad que estaba buscando o la hallara en los dáctilos ideos en una de sus reencarnaciones anteriores ${ }^{32}$, lo cierto es que su descubrimiento alteró el curso de la ciencia matemática y abrió el camino de lo que ahora conocemos como «física acústica». Así, inspiración divina aparte, el hallazgo de la verdad acústica fue para el maestro de Samos de instinto esen-

o $\tilde{\eta} \theta$ oc, Heraclides Póntico (ap. Ath. XIV 19.16-19) la define como varonil y majestuosa ( $\dot{\alpha} v \delta \rho \tilde{\omega} \delta \varepsilon \varsigma$,

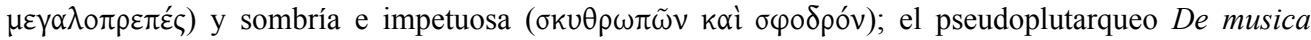

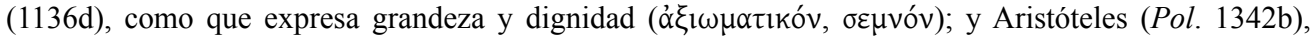
como la más firme y de carácter varonil. Sobre este modo y sobre el carácter musical, vid. MichAELIDES (1978: s. v. «dorios», «ethos» $\mathrm{y}$ «harmonia»). Esta harmonía, junto con la frigia, resultó ser aceptada por Platón (R. 399a-c) por ser apta y estar adaptada a los guerreros y a los hombres prudentes. Según Dionisio Yambo (ap. Ps. Plu. De musica, 1136c), esta harmonía fue inventada por Torebo, un músico mítico conocido también como Tirreno que, además, añadió la quinta cuerda a la lira.

${ }^{28}$ Cf. Iamb. VP. 35.110.

${ }^{29}$ Cf. Phlb. 56a3-c6. Las herramientas platónicas comunes a las mencionadas por Nicómaco son la

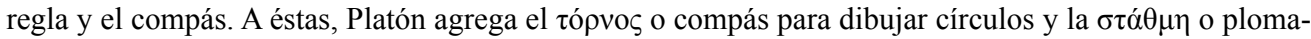
da. Podemos imaginar que el filósofo de Atenas desconocía la «ayuda instrumental auditiva» que, según cuenta nuestro autor, inventó Pitágoras en beneficio de la exactitud. Cf. LEvin (1994:86-97).

${ }^{30} \mathrm{Cf}$. Ptol. Harm. I 1-2, Düring:3-6, en donde trata del oído y de la razón como criterios de la harmónica frente a los demás sentidos y, a su vez, del llamado «canon» como herramienta propia del estudio de ésta.

${ }^{31}$ R. 531a4-b4. En este pasaje Glaucón hace mofa de aquellos músicos que, para hallar el intervalo medible más pequeño, debían afinar sus cuerdas con puras conjeturas, anteponiendo sus oídos a sus mentes, «provocando tormentos a las cuerdas y torturándolas al estirarlas sobre las clavijas».

${ }^{32}$ Así lo cree Levin (1994:89). Téngase en cuenta, asimismo, que la doctrina de la metempsicosis (DRAE: «doctrina religiosa y filosófica de varias escuelas orientales, y renovada por otras de Occidente, según la cual las almas transmigran después de la muerte a otros cuerpos más o menos perfectos, conforme a los merecimientos alcanzados en la existencia anterior») es muy fuerte en los diversos relatos de la vida de Pitágoras. $C f$. D.L. VIII 4. 
cialmente mental, pues éste se obtiene utilizando el intelecto (voũ $\varsigma$ ) y no por medio de los sentidos (åkoń), lo que demuestra su superioridad. Sin embargo, este tipo de conocimiento «mental» debía de estar precedido de pruebas empíricas ${ }^{33}$. Por eso, según cuenta Nicómaco, luego de descubrir que el tono del sonido producido por cada martillo dependía exclusivamente de la masa o el peso de éstos, sin intervenir en ningún caso ni el impacto o la fuerza de golpeo, ni la altura de caída, ni el hierro que se estaba forjando, ni el yunque ni la forma de los martillos, Pitágoras trasladó el experimento a su casa, cambiando los martillos por cuatro pesos colgados de cuerdas suspendidas en

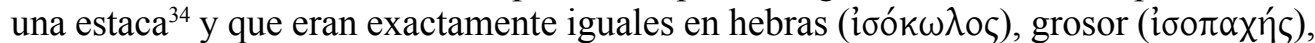

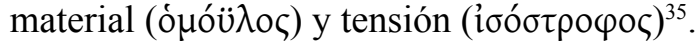

En cada uno de los extremos de estas cuatro cuerdas Pitágoras colocó un peso e igualó las cuerdas en longitud. Luego las golpeó a la vez de dos en dos y, al hacerlo, observó que ocurría lo mismo que en la herrería: dependiendo de la relación de masas que colgaban de cada cuerda y, por tanto, de su tensión, el sonido producido respondía a intervalos de cuarta, quinta y octava. Concluyó, así, que los pesos que determinaban las variaciones de los sonidos que estaba escuchando eran los que tenían los valores de 12, 6, 8 y 9, a partir de lo cual definió las diferentes relaciones musicales: epítrita o relación de cuarta, que se da entre la cuerda con 8 pesos y la de 6 (3:4); hemiólica o relación de quinta, que se da entre la cuerda con 9 pesos y la de 6 (2:3); epogdoica o relación de tono, entre la cuerda con 9 pesos y la de 8 (9:8); doble o relación de octava, entre la cuerda con 12 pesos y la de $6(2: 1)$.

De este modo Pitágoras pudo demostrar - según cuenta Nicómaco- que la octava resulta de la combinación $(\sigma u ́ \sigma \tau \eta \mu \alpha)$ conjunta ( $\dot{\varepsilon} v \sigma v v \alpha \varphi \tilde{n})$ de una quinta $(3: 2)$ y una cuarta (4:3), o de una cuarta (4:3) y una quinta (3:2) (imagen 5).

Los números $6,8,9$ y 12 no son elegidos por azar, pues 6 es el primer número cuyas relaciones $4: 3$, 3:2 y 2:1 son números enteros, dando como resultado el resto de los números citados ( 8,9 y 12) (imagen 6$)$.

8. El análisis de este pasaje evidencia una serie de «faltas» tanto para la tensión de las cuerdas como para la percusión de los yunques. La justificación de la aparición de estos descuidos se debe a que Nicómaco obvió poner en práctica el experimento que él mismo estaba describiendo para evitar encontrarse con serias dificultades inherentes en su realización. Estos fallos fundamentales pueden resumirse en lo que podríamos llamar «error de percusión» y «error de tensión». El primero de ellos demuestra que la variación tonal producida por la percusión depende del objeto golpeado (el yunque) y de sus características físicas más que de los objetos que golpean (los martillos). A modo de comparación, podemos pensar en címbalos, campanas o platos de metal, cuya forma (tamaño y grosor, principalmente) determinarán su variación tonal ${ }^{36}$. Así,

${ }^{33}$ Así Fideler (1988:47).

${ }^{34}$ Sobre esta barra puesta de pared a pared, formando un ángulo, vid. Meibomius (1652:47, s. $v$

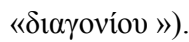

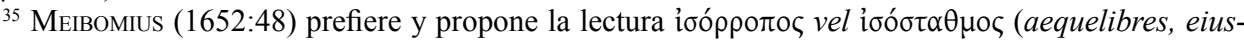
dem ponderis).

${ }^{36} C f$. Wood (1944:176-177). En estas páginas, el autor plasma sus reflexiones sobre las complejidades vibratorias de estos instrumentos. 
Pitágoras habría necesitado cuatro yunques en su comprobación y no uno sólo golpeado por cuatro martillos y del que salen emitidos cuatro tonos diferentes ${ }^{37}$. Casi al final del capítulo, Nicómaco cuenta que Pitágoras «extendió el experimento», entre otros instrumentos, a platos de metal, a partir de los cuales sí que podría haber descubierto el principio más básico sobre la importancia de la percusión en la producción de sonido. De cualquier manera, sabemos de otros experimentos similares capitaneados por un seguidor y contemporáneo de Pitágoras: Hipaso de Metaponto ${ }^{38}$. Según Aristóxeno ${ }^{39}$, Hipaso produjo las consonancias de cuarta, quinta y octava usando cuatro discos de bronce de igual diámetro, pero con grosor distinto, pues uno tenía la proporción 4:3, otro la proporción $3: 2$ y otro la proporción 2:1, respectivamente. Añade Aristóxeno que Glauco de Regio, músico y musicólogo pitagórico que vivió entre el siglo v y IV a. C., luego de conocer estos cuatro discos «afinados» de Hipaso, pudo tocar una melodía con ellos ${ }^{40}$. La historia transmitida por el tarentino es corroborada por Teón de Esmirna, que refiere, además, otro experimento de la mano de Laso de Hermíone. Éste consistía en la percusión de unos vasos huecos rellenos de distintas cantidades de agua ${ }^{41}$.

Aunque el primero en detectar el error del experimento de Pitágoras fue el matemático francés Jean-Etienne Montucla (1725-1799) en Histoire des Mathematiques y aun habiendo considerado la tradición a Sir Isaac Newton (1643-1727) como pionero en hallar el «error de tensión» latente en el relato de Nicómaco ${ }^{42}$, se acepta que no se descubrió como tal hasta el siglo XIX con la figura de Théodore H. Martin ${ }^{43}$. El estudioso francés mostró que las proporciones en las que Pitágoras se basó, según Nicómaco, no podían expresar de manera exacta la relación entre un tono de la cuerda y la cantidad de tensión necesaria para producir las vibraciones tonales.

Así, para la relación entre tensión y frecuencia de vibración, ésta ha de ser la raíz cuadrada de aquélla, lo que implica que, para producir una octava en una cuerda, su tensión debe ser cuatro veces mayor que la que produce la nota más baja, de la misma manera que, para producir una quinta, se necesita una tensión dos y cuatro veces mayor, y una cuarta, una tensión una y siete novenos mayor ${ }^{44}$. Es decir: a igualdad de longitud y del tipo de cuerda, la frecuencia es directamente proporcional a la raíz cuadrada del peso tensor (imagen 7).

${ }^{37}$ GUTHRIE (2005:217): «golpear una pieza de hierro sobre un yunque con martillos de pesos diferentes produce una diferencia exigua o ninguna en el tono de los sonidos».

${ }^{38}$ Curiosamente este Hipaso fue el primero en demostrar la irracionalidad de $\sqrt{2}$.

${ }^{39}$ Fr. 90.

${ }^{40} C f$. in Phd. 108d, Diels-Kranz:8.12, donde se recogen estas dos noticias a partir de la expresión

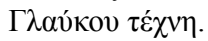

${ }^{41}$ Theo Sm. LIX 4-21.

${ }^{42}$ En Scholia de Principia mathematica, II 4-9, Sir Isaac Newton no menciona ni el nombre de Nicómaco ni su tratado harmónico, pero la explicación al fallo del experimento de Pitágoras evoca, sin lugar a dudas, el nombre y la obra musical nicomaquea. La línea argumental newtoniana se basa en la nula observación pitagórica de la llamada «Ley del cuadrado inverso», adjunta a la Ley de la Gravitación Universal.

${ }^{43} C f$. (1841:391), donde, extrañamente, no nombra a Nicómaco, sino a los transmisores de la leyenda de Pitágoras y los martillos, comenzando por Gaudencio el Filósofo.

${ }^{44}$ Cf. Chaignet (1873:134), Jan (1895:141), Burnet (1957:106), VAn Der Waeden (1943:179ss.), GUTHRIE (2005:217-219) y BURKERT (1972:375-376). 
En definitiva, los intervalos medidos según el experimento de Pitágoras quedaban considerablemente cortos para una octava, una quinta y una cuarta, pues, según lo dicho, las auténticas relaciones que los expresan son, respectivamente, 4:1, 9:4 y 16:9.

Asimismo, es menester tener presente que, pese a la aceptación generalizada de que Marin Mersenne ${ }^{45}$ descubrió en el siglo XVII la relación entre tensión y frecuencia de vibración, hay pruebas de que Claudio Tolomeo se adelantó al musicólogo francés en siglos; de hecho, fue el primero que intentó repetir tal cual el experimento descrito por Nicómaco ${ }^{46}$. En su tratado harmónico, el alejandrino da dos razones para justificar el fracaso de las pruebas sobre las tensiones mediante pesos: por un lado, la dificultad de hallar segmentos de cuerda exactamente iguales en densidad y espesor y, por otro lado, la imposibilidad de evitar que el peso, al extenderlos, modifique su originaria densidad. Los martillos y percusiones presentan, asimismo, un obstáculo en su material y forma porque no es posible mantenerlos sin alterar. Por último, los aerófonos también se ven afectados por una traba que consiste en la complejidad y dificultad que implica establecer la extremidad para medir la longitud de la embocadura.

En cuanto al «error de tensión», la inexactitud del geraseno esté, quizá, en utilizar la relación entre tensiones de cuerdas de la misma longitud, en vez de utilizar la de las longitudes de cuerdas sometidas a una misma tensión. Es decir, si el experimento se realiza con cuatro cuerdas de longitudes correspondientes a las relaciones mencionadas, tensadas con la misma intensidad (por ejemplo, atándolas a una misma barra inclinada), los sonidos resultantes son, en efecto, los de cuarta, quinta y octava, ya que la relación entre frecuencias del sonido y longitudes de las cuerdas que los producen son inversamente proporcionales ${ }^{47}$. De hecho, los actuales instrumentos de cuerda con mástiles usan este principio de variación de longitud de una misma cuerda para producir las distintas notas, cuando el músico presiona cada cuerda contra el mástil, acortando así su longitud inicial (imagen 8).

9. Lo que aquí se relata, pues, no tiene fundamento físico real, dado que la relación existente entre tensiones o masas y sonidos, que no son sino las existentes entre las longitudes de onda sonora, no es aritmética, sino que está en función de la raíz cuadrada de las primeras ${ }^{48} \mathrm{y}$, además, cuando un martillo golpea un yunque, el cuerpo que

${ }^{45}$ Fraile minorita francés del siglo XVII (1588-1648) que se dedicó al estudio de diversos campos, como la filosofía, la teología, las matemáticas y la teoría musical.

${ }^{46} \mathrm{Cf}$. Harm. I 8, Düring:17.15-16. Tolomeo, luego de usar pesos para tensar las cuerdas, concluye reconociendo la existencia de un aumento en el tono, pero no coincidente con las proporciones de los pesos. BARKER (1989:258, n. 49) considera que tanto la explicación como las conclusiones a las que llega el astrónomo y matemático alejandrino, pese a tener algo de verdad, no vienen al caso, afirmación con la que discrepamos. La descripción de procedimientos similares tolemaicos la hallamos, asimismo, en Harm. III 1-2, Düring:83-91. Vid. Levin (1980:228-9).

${ }^{47} \mathrm{Cf}$. la teoría de la proporcionalidad inversa de Marin Mersenne. Las leyes a las que nos referimos aparecen recogidas en su Traité de l'harmonie universelle y podrían resumirse de la siguiente manera: la frecuencia del sonido producido por una cuerda es inversamente proporcional al doble de su longitud; es directamente proporcional a la raíz cuadrada de la tensión a la que está sometida; es inversamente proporcional a la raíz cuadrada de su densidad; y es inversamente proporcional a la raíz cuadrada de su sección (área transversal).

${ }^{48}$ Así también Mathiesen (1998:74, n. 20). 
vibra y, por tanto, produce el sonido es el yunque y no el martillo. La historia sería físicamente verosímil si se golpearan tres yunques de distinta masa con el mismo martillo o si se tañeran tres cuerdas de longitudes diferentes sometidas a la misma tensión ${ }^{49}$.

10. En conclusión, todos estos elementos (los martillos de la herrería, los pesos en los extremos de las cuerdas y sus supuestos equivalentes, a saber, las tensiones en las cuerdas) forman parte de lo fabuloso de una de las grandes leyendas de todos los tiempos; pero, como en toda leyenda, su objeto se centra en la noción de la materialidad del número, fundamento principal del pensamiento pitagórico. El influjo y expansión del pitagorismo impulsó a los seguidores del de Samos a franquear la frontera del número, entendido ahora como sustancia material, para «construir el mundo», un concepto que, a más que abstracto, fue enfatizado por nuestro autor en las diversas referencias al peso en este capítulo ${ }^{50}$.

11. Al final del relato se nos cuenta que Pitágoras cambió la configuración del experimento, pasando de cuerdas de las que suspendía pesos a cuerdas tensadas mediante clavijas, con una relación entre las tensiones aplicadas equivalente a los pesos anteriormente citados. Así, tras probar insistentemente su primer artilugio de cuatro

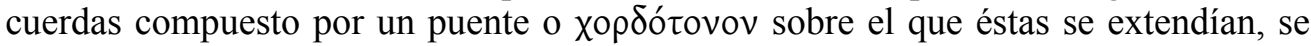
ataban y liaban sobre sí mismas en clavijas, reproduciendo una tensión equivalente a la de aquellos pesos, el de Samos lo perfeccionó hasta lo que se ha reconocido como la herramienta acústica pitagórica por excelencia: el monocordio ${ }^{51}$. Comparando el

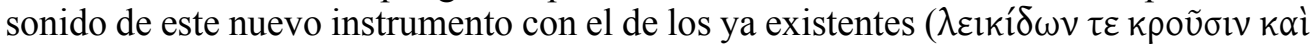

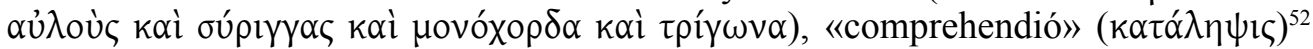
que la relación numérica entre masas, dimensiones, tensiones y notas era equivalente a la anteriormente descrita. Es decir: las cuerdas sometidas a la menor tensión producían los tonos más bajos, mientras que los tonos agudos resultaban de las cuerdas sometidas a la mayor tensión.

${ }^{49}$ Según PeirCe ap. Eisele (1985.I:216-226), el error puede venir provocado por el carácter secreto de los estudios del maestro, que conseguía confundir a muchos de sus discípulos.

${ }^{50} \mathrm{He}$ aquí el léxico empleado por nuestro autor: őүкоৎ (sólo este término fue aceptado por los

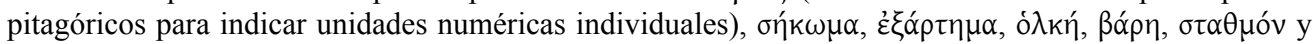
ßpĩ日os.

${ }^{51}$ Cf. D.L. VIII 12 y Gaud. Harm. XI, Jan:340.4-341.25. Recuérdese que Aristid. Quint. III 2, W-I:97.3-4 cuenta que Pitágoras aconsejaba a sus discípulos el manejo de este instrumento para la investigación acústica. Para un estudio mucho más detallado del monocordio, vid. BURKERT (1972:375, n. 22) y Fideler ap. Guthrie (1988:24-26).

${ }^{52}$ Este término técnico es propio del pensamiento estoico e indica un momento del proceso cognitivo que implica el ascenso de la razón a la representación, dando lugar a la comprensión del objeto por parte del sujeto. Cf. SVF I 60-68; Nicom. Ar. I 1; Stob. Ecl. II 73 y 111; S.E. M. VII 151; y D.L. VII 47. 


\section{BibLIOGRAFÍA}

BArker, Andrew (1989), Greek Musical Writings, vol. II, «Harmonic and Acoustic Theory», Cambridge: Cambridge University Press.

Burkert, Walter (1972), Lore and Science in Ancient Pythagoreanism, Cambridge: Harvard University Press.

Burnet, John (1957), Early Greek Philosophy, New York.

Chaignet, Anthelme-Édouard (1873), Pythagore et la Philosophie Pythagoricienne, vol. II, Paris.

D’Ooge, Martin Luther, Robbins, Frank E. and Karpinski, Louis C. (1926), Nicomachus of Gerasa. Introductio to Arithmetic, New York.

Fideler, David R. ap. Guthrie, Kenneth S. (1988), The Pythagorean Sourcebook and Library: An Anthology of Ancient Writings which Relate to Pythagoras and Pythagorean Philosophy, Grand Rapids: Phanes Press.

Flannery, David (2005), The Square Root of Two, Springer.

Fowler, David y RoBson, Eleanor (1998), «Square Root Approximations in Old Babylonian Mathematics: YBC 7289 in Context», HM 25.4:366-378.

FrANK, Erich (1923), Plato und die sogenannten Pythagoreer. Ein Kapitel aus der Geschichte des griechischen Geistes, Halle.

Guthrie, William Keith C. (2005), Historia de la filosofía griega, vol. I, Barcelona: RBA (versión española de Medina González, Alberto (1971) de A History of Greek Philosophy, vol. I, The Earlier Presocratics and the Pythagoreans, Cambridge).

Levin, Flora Rose (1975), The Harmonics of Nicomachus and the Pythagorean Tradition, American Classical Studies, no. 1 University Park, The American Philological Association.

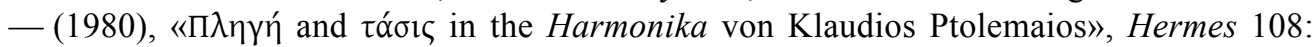
205-229.

- (1994), Nicomachus. Manual of Harmonics. Translation and Commentary, New York.

MacDonald Cornford, Francis (1937), Plato's Cosmology. The Timaeus of Plato translated with a running commentary, London.

Martin, Théodore H. (1841), Études sur le Timée de Platon, Paris.

Mathiesen, Thomas J. (ed.) (1998), Strunk's Source Readings in Music History, vol. I, Greek View of Music, New York.

McDermott, William C. (1977), «Plotina Augusta and Nicomachus of Gerasa», Historia 26: 192-203.

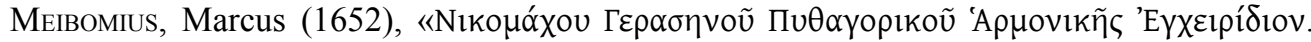
Nicomachi Geraseni Pythagorici Harmonices Manuale. Marc. Meibomius primus vertit, ac notis explicavit», Antiquae musicae auctores septem. Graece et Latine. Marcus Meibomius restituit ac notis explicavit, Amstelodami, I-II vol. Apud Ludovicum Elzevirium.

Meriani, Angelo (1995), «Un esperimento di Pitagora (Nicom. Harm. Ench. 6, pp. 245-248 Jan)», MOUSIKE. Metrica ritmica e musica greca in memoria di G. Comotti, a cura di B. Gentili e F. Perusino, Istituti Editoriali e Poligrafici Internazionali (Studi di metrica classica, 11), Pisa-Roma: 77-92.

Michaelides, Solon (1978), The Music of Ancient Greece. An Encyclopaedia, London.

Neugebauer, Otto (1957), The Exact Sciences in Antiquity, New York. 
Peirce, Charles S. ap. Eisele, Charles S. (ed.) (1985), Historical Perspectives on Peirce's Logic of Science. A History of Science, 2 vols., Berlin.

Philip, James A. (1966), Pythagoras and Early Pythagoreanism, Toronto.

REDONDO REYEs, Pedro (2005), «Pitágoras en la herrería: variaciones sobre un episodio legendario», Prometheus 31.3:193-215.

Ruelle, Charles Emile (1880), Collection des autres grecs relatifs à la musique, vol. II, Nicomaque de Gérase. Manuel d'Harmonique, Paris.

VAn der Waeden, Bartel Leendert (1943), «Die Harmonielehre der Pythagoreer», Hermes 78:163-199.

Von JAn, Karl (1895), Musici scriptores Graeci (MSG). Aristoteles, Euclides, Nicomachus, Bacchius, Gaudentius, Alypius et melodiarum veterum quidquid exstat. Recognovit prooemiis et indice instruxit Carolus Janus. Annexae sunt tabulae.

WERnER, Eric (1959), The Sacred Bridge, London-New York.

Wood, Alexander (1944), The Physics of Music, London.

ZELLER, Eduard (19196), Die Philosophie der Griechen in ihrer geschichten Entwickung, vol. I, Leipzig. 


\section{APÉNDICE DE IMÁGENES}

$1+\frac{24}{60}+\frac{51}{60^{2}}+\frac{10}{60^{3}}=1,41421 \overline{296}$

Imagen 1: aproximación de $\sqrt{2}$

$$
1+\frac{24}{60}+\frac{84}{60}=\frac{4}{3}
$$

Imagen 2: dos primeros sumandos de la aproximación de $\sqrt{2}$

$$
\frac{3}{2}-\frac{4}{3}=\frac{9}{8}
$$

Imagen 3: implicación de $\sqrt{2}$ en la relación tonal o epogdoica

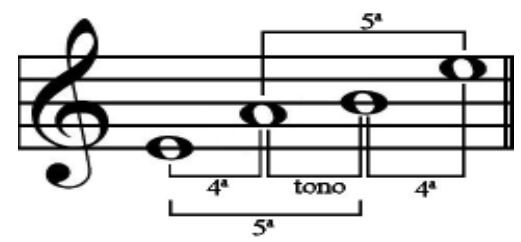

Imagen 4: lo que Pitágoras escuchó en la herrería

$$
\begin{aligned}
& 12: 8: 6, \text { o mejor } \frac{12}{8} \times \frac{8}{6}=\frac{96}{48}=\frac{2}{1} \\
& 12: 9: 6, \text { o mejor } \frac{12}{9} \times \frac{9}{6}=\frac{108}{54}=\frac{2}{1}
\end{aligned}
$$

Imagen 5: combinaciones para hallar la octava 


\begin{tabular}{|c|c|c|c|}
\hline $\mathbf{N .}^{\mathbf{0}}$ & $\mathbf{N .}^{\mathbf{0}} \times \mathbf{4 : 3}$ & $\mathbf{N}^{\mathbf{}} \times \mathbf{3 : \mathbf { 2 }}$ & $\mathbf{\mathbf { N }}{ }^{\mathbf{}} \times \mathbf{2 : \mathbf { 1 }}$ \\
\hline 1 & 1,34 & 1,50 & 2 \\
\hline 2 & 2,63 & 3 & 4 \\
\hline 3 & 4 & 4,5 & 6 \\
\hline 4 & 5,34 & 6 & 8 \\
\hline 5 & 6,67 & 7,5 & 10 \\
\hline $\mathbf{6}$ & $\mathbf{8}$ & $\mathbf{9}$ & $\mathbf{1 2}$ \\
\hline
\end{tabular}

Imagen 6: números 6, 8, 9 y 12 y su relación con los intervalos de cuarta, quinta y octava

$$
f=\frac{\sqrt{t: \rho}}{2 l}
$$

( $f$ es la frecuencia, $t$ la tensión, $\rho$ la masa y $l$ la longitud)

Imagen 7: relación entre tensión y frecuencia de vibración

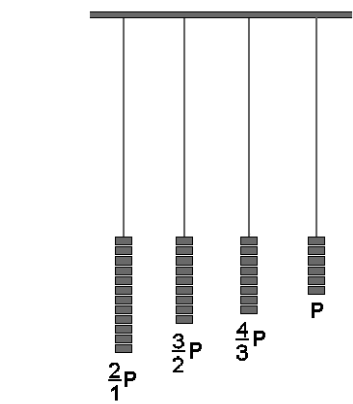

Experimento de Pitágoras según Nicómaco Misma longitud, diferentes tensiones

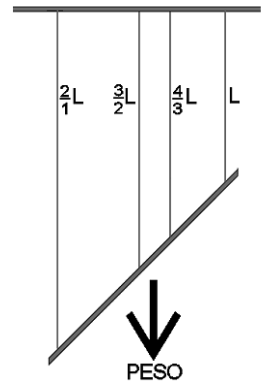

Posibilidad más real. Misma tensión, diferente longitud

Imagen 8: error de tensión 